\title{
Long noncoding RNA MIR99AHG promotes gastric cancer progression by inducing EMT and inhibiting apoptosis via miR577/FOXP1 axis
}

Qingyang Meng ${ }^{1 \dagger}$, Xiangjun Wang $^{2 \dagger}$, Tongqing Xue ${ }^{3}$, Qiangfang Zhao ${ }^{3}$, Wei Wang ${ }^{3}$ and Kun Zhao ${ }^{3 *}$ (e)

\begin{abstract}
Background: Long non-coding RNAs (IncRNAs) play a vital role in the genesis and development of human cancer. LncRNA MIR99AHG has been reported to be upregulated in acute myeloid leukemia (AML); however, its function in gastric cancer (GC) is still not clear. Here we were aiming to screen the prognostic IncRNA candidates and to explore the function of MIR99AHG in GC.

Methods: We have preliminarily screened some candidate IncRNA biomarkers in GC tissues through analyzing microarray datasets. The expression level of MIR99AHG in GC cell lines and tissues was monitored via qPCR. Survival analysis was performed with the patients of our hospital and TCGA database cases. CCK-8 assay, trans-well assay and flow cytometry were performed to determine cell proliferation, invasion, migration and apoptosis. Meanwhile, a target of MIR99AHG was predicted and identified by luciferase reporter gene detection experiments.

Results: MIR99AHG was strongly up-regulated in human GC and contributed to cancer progression. Kaplan-Meier analysis revealed that up-regulating MIR99AHG expression was positively correlated with unfavorable overall survival $(P<0.01)$ of patients from our hospital and TCGA database. Knockdown of MIR99AHG expression inhibited cell proliferation, invasion, migration and promoted cell apoptosis. Moreover, MIR99AHG worked as an oncogenic gene though competing for endogenous RNA (ceRNA) of miR-577.
\end{abstract}

Conclusions: Our findings suggested that MIR99AHG contributes to malignant phenotypes of GC and may become a promising therapeutic target.

Keywords: Gastric cancer, MIR99AHG, miR-577, IncRNA, ceRNA, miRNA, FOXP1

\section{Background}

Gastric cancer (GC) is the leading cause of cancer-related mortality worldwide [1]. The genesis and progression of GC is an intricate process involving numerous coding and non-coding genes [2]. Surgery is still the optimal treatment for GC patients. The survival of GC patients

\footnotetext{
*Correspondence: zhaokuntll@163.com

${ }^{\dagger}$ Qingyang Meng and Xiangjun Wang contributed equally and should be regarded as co-first authors

${ }^{3}$ Department of Oncology, Huaian Hospital of Huaian City, No.161 Zhenhuailou East Road, Huai'an 223200, Jiangsu, China

Full list of author information is available at the end of the article
}

has been improved with the development of early detection and radical surgery [3]. However, the prognosis of GC patients remains not optimistic despite advances in surgical techniques, chemotherapy, radiotherapy and targeted therapy [4]. Therefore, identifying valuable therapeutic targets is extremely important for the GC treatment [5].

Long non-coding RNAs (lncRNAs) are characterized as transcripts of over 200 nucleotides in length, which have been demonstrated to play pivotal roles in cancer development [6]. It is known that lncRNAs were widely transcribed in the genome, but our understanding of their functions was limited [7]. Notably, accumulative evidence 
disclosed the indispensable involvement of lncRNAs in human malignancies in either pro-tumoral or anti-tumor manners [8].

The MIR99AHG is located on chromosome 21q21.1 and transcribed as a polycistronic primary transcript that produces a spliced lncRNA and three intronic microRNAs (miRNAs): MIR99A, MIR125B2, and LET7C (MIRLET7C). The IncRNA MIR99AHG has a role in cell proliferation and differentiation. Emmrich et al. reported that MIR99AHG, which they called MONC, showed significantly higher expression in acute megakaryoblastic leukemia (AMKL) cell lines. The knockdown of MIR99AHG impeded the proliferation of AMKL cell lines and patient-derived samples [9]. However, whether this lncRNA correlates with GC is currently unknown; therefore, here we first analyzed MIR99AHG expression levels in GC tissues and evaluated the relationship between the expression levels and the clinicopathological features of GC patients. Then experiments were carried out to detect the function of MIR99AHG in GC and the underlying mechanisms.

\section{Methods}

\section{Bioinformatical analysis}

The human microarray datasets (GSE109476) were downloaded from Gene Expression Omnibus (GEO) database and background adjusted by using Robust Multichip Average. Limma package was applied to analyze microarrays for gene expressions of lncRNAs. The Cancer Genome Atlas (TCGA) data were downloaded from the GDC portal. EdgeR package was applied to analyze TCGA data on a local computer for gene expression.

\section{Tissue samples and clinical data collection}

In this research, we analyzed 118 patients who underwent resection of the primary GC at the Zhongshan Hospital, Fudan University, and Shanghai, China. This study was approved by the Ethics Committee on Human Research of the Zhongshan Hospital, Fudan University, Shanghai, China and all the patients have signed out the written informed consent. The clinicopathological information of all the patients was listed in Table 1. All the patients have been undergoing follow-up until November 2018, and the median follow-up period was 36 months (range 20-48 months). OS was defined as the interval between the surgery time and death time. The specimens were treated following the ethical standards. This study was conducted in accordance with the Declaration of Helsinki.

\section{Cell culture}

Human GC cell lines SGC7901, BGC823, MGC803, AGS and MKN45 and the normal gastric epithelium cell line
(GES-1) were purchased from the Chinese Academy of Sciences Committee (Shanghai, China). MGC803, AGS and BGC823 cells were cultured in RPMI 1640; MKN45, GES-1 and SGC7901 cells were cultured in DMEM (GIBCO-BRL) medium supplemented with $10 \%$ fetal bovine serum (FBS; Gibco Life Technologies, Gaithersburg, MD), 1\% penicillin and streptomycin (Gibco Life Technologies). The cells were cultured at $37{ }^{\circ} \mathrm{C}$ in $5 \%$ $\mathrm{CO}_{2}$.

\section{RNA preparation and quantitative real-time $P C R$}

Total RNA was extracted from cells or tissues with TRIzol reagent (Invitrogen, USA) following the protocol. RNA was qualified using a NanoDrop spectrophotometer and the A260/A280 ratio was used to test the RNA purity. Total RNA was converted to cDNA by reverse transcription and then quantitative real-time PCR (qRT-PCR) was carried out using the commercial kit (Invitrogen, USA). Relative transcript expression levels were calculated by the comparative $2^{-\Delta \Delta \mathrm{CT}}$ method normalize to GAPDH.

\section{CCK-8 assay}

Cell Counting Kit-8 (Sigma, Japan) was applied to determine the cell viability of GC cells. Cells were seeded in 96-well plates at $1 \times 10^{4}$ cells per well and cultured for $24 \mathrm{~h} .10 \mu \mathrm{L}$ of the cell proliferation reagent CCK-8 to each well and mixed then incubated for $90 \mathrm{~min}$ at $37{ }^{\circ} \mathrm{C}$. The optical density (OD) of each well was detected at wavelength of a $450 \mathrm{~nm}$.

\section{Cell migration and invasion assays}

Cell migration and invasion assay were carried out using transwell chambers (8- $\mu \mathrm{m}$ pore size; Millipore, Bedford, MA, USA) coated without/with Matrigel (Sigma-Aldrich, St. Louis, MO, USA). After transfecting for $48 \mathrm{~h}$, cells suspended in serum-free media were seeded in the upper transwell chamber. The lower chamber was then added with medium containing 10\% FBS. After incubation for $24 \mathrm{~h}$, cells had migrated or invaded through the membrane were fixed with formaldehyde and stained with $0.1 \%$ crystal violet after wiping out cells remaining on the upper membrane. Then cells were imaged and counted with the microscope. Independent experiments were carried out for 3 times.

\section{Western blotting analysis}

Cells were lysed with RIPA lysis buffer and total protein was extracted and quantified with the BCA kit (Beyotime, Beijing, China). The sodium dodecyl sulfate-polyacrylamide gel electrophoresis (SDS-PAGE) was used to separate $20 \mu \mathrm{g}$ of protein extract and polyvinylidene fluoride (PVDF) membrane (Millipore) was used for 
Table 1 Correlation between MIR99AHG expression and clinicopathological characteristics of GC

\begin{tabular}{|c|c|c|c|}
\hline \multirow[t]{2}{*}{ Clinical parameter } & \multirow{2}{*}{$\begin{array}{l}\text { MIR99AHG } \\
\text { High no. cases }(n=59)\end{array}$} & \multirow{2}{*}{$\begin{array}{l}\text { Expression } \\
\text { Low no. cases }(n=59)\end{array}$} & \multirow[t]{2}{*}{ P-value } \\
\hline & & & \\
\hline Age (years) & & & 0.732 \\
\hline$\leq 50$ & 31 & 36 & \\
\hline$>50$ & 28 & 23 & \\
\hline Gender & & & 0.554 \\
\hline Male & 37 & 33 & \\
\hline Female & 22 & 27 & \\
\hline Location & & & 0.679 \\
\hline Distal & 20 & 24 & \\
\hline Middle & 21 & 23 & \\
\hline Proximal & 18 & 12 & \\
\hline Size $(\mathrm{cm})$ & & & 0.654 \\
\hline$>5$ & 35 & 33 & \\
\hline$\leq 5$ & 29 & 31 & \\
\hline Histologic differentiation & & & 0.008 \\
\hline Well & 9 & 13 & \\
\hline Moderately & 17 & 20 & \\
\hline Poorly & 21 & 10 & \\
\hline Undifferentiated & 12 & 16 & \\
\hline Invasion depth & & & $<0.001$ \\
\hline $\mathrm{T} 1$ & 4 & 15 & \\
\hline $\mathrm{T} 2$ & 10 & 21 & \\
\hline $\mathrm{T} 3$ & 20 & 15 & \\
\hline T4 & 15 & 8 & \\
\hline TNM stages & & & 0.003 \\
\hline$|-| \mid$ & 20 & 35 & \\
\hline III-IV & 39 & 24 & \\
\hline Lymphatic metastasis & & & $<0.001$ \\
\hline Yes & 39 & 23 & \\
\hline No & 20 & 36 & \\
\hline Distant metastasis & & & 0.02 \\
\hline Yes & 15 & 7 & \\
\hline No & 44 & 52 & \\
\hline
\end{tabular}

transferring the protein from the gel. GAPDH was applied for normalization.

\section{Flow-cytometric analysis}

Cells were double-stained with fluorescein isothiocyanate (FITC)-Annexin $\mathrm{V}$ and propidium iodide was done by the FITC Annexin V Apoptosis Detection Kit (Nanjing KeyGen Biotech. Co. Ltd., China). Following the manufacturer's protocol. Cell apoptosis was analyzed by using Cell Quest software on a FACSAria Flow Cytometer (BD Inc., USA). Fluorescence was detected with an excitation wavelength of $480 \mathrm{~nm}$. Cells were analyzed with FACSAria Flow Cytometer (BD Biosciences, USA).

\section{Gene transfection}

Overexpressed plasmids and siRNAs together with the negative controls (GenePharma, China) were transfected into cells according to the manufacturer's protocol of the Lopfectamine 2000 (Invitrogen, USA).

\section{Dual-luciferase reporter assay}

The mutant type and wild type of luciferase reporter vector targeting MIR99AHG or FOXP1 binding sites were synthesized (Promega, USA). Luciferase activities were detected after transfection for $48 \mathrm{~h}$. Renilla luciferase activity was used for normalization. 


\section{Statistical analysis}

All statistical analyses were carried out with SPSS v.20.0 (SPSS) and GraphPad Prism 7.0. Experiments were repeated for at least 3 times and Student's -test and oneway ANOVA were mostly performed. Survival curves were plotted using the Kaplan-Meier method and with log-rank tests comparison, Survival data were analyzed with univariate and multivariate Cox proportional hazards models. Two-tailed $P$ values were applied with a value of $\mathrm{P}<0.05$ as significant results.

\section{Results}

MIR99AHG upregulation was associated with aggressive clinical characteristics and unfavorable prognosis of GC

To explore the significant lncRNAs in GC, 5 paired global expression profiles of lncRNAs in GC and adjacent tissues were downloaded from the GEO database (GSE109476). There were 355 unregulated lncRNAs showed obvious fold changes in cancer group compared to adjuvant normal tissues, $\log \mathrm{FC}>1$ and $\operatorname{padj}<0.01$ (Fig. 1a). Among them, the function of MIR99AHG in GC was not defined. The median MIR99AHG expression levels in the GC tissues were 2.4 times higher than those in the adjuvant normal tissues (Fig. 1b). Then MIR99AHG expression levels were also investigated in the patients from the TCGA database, the MIR99AHG expression level was higher and showed broader ranging in GC group compared with normal group (Fig. 1c). Kaplan-Meier analysis and log-rank test were carried out to investigate the relationship between MIR99AHG expression and the clinicopathological features on overall survival (OS). Firstly, the OS was explored in the 118 paired samples, the results showed that the MIR99AHG high group had inferior overall survival (median OS: 16 months) than low group (median OS: 23 months; $\mathrm{P}<0.01$ ) (Fig. 1d). The 3 -years OS rates were $22.4 \%$ (16.9-27.7\%) in the MIR99AHG high group and 39.1\% (33.9-45.9\%) in the MIR99AHG low group. Univariate

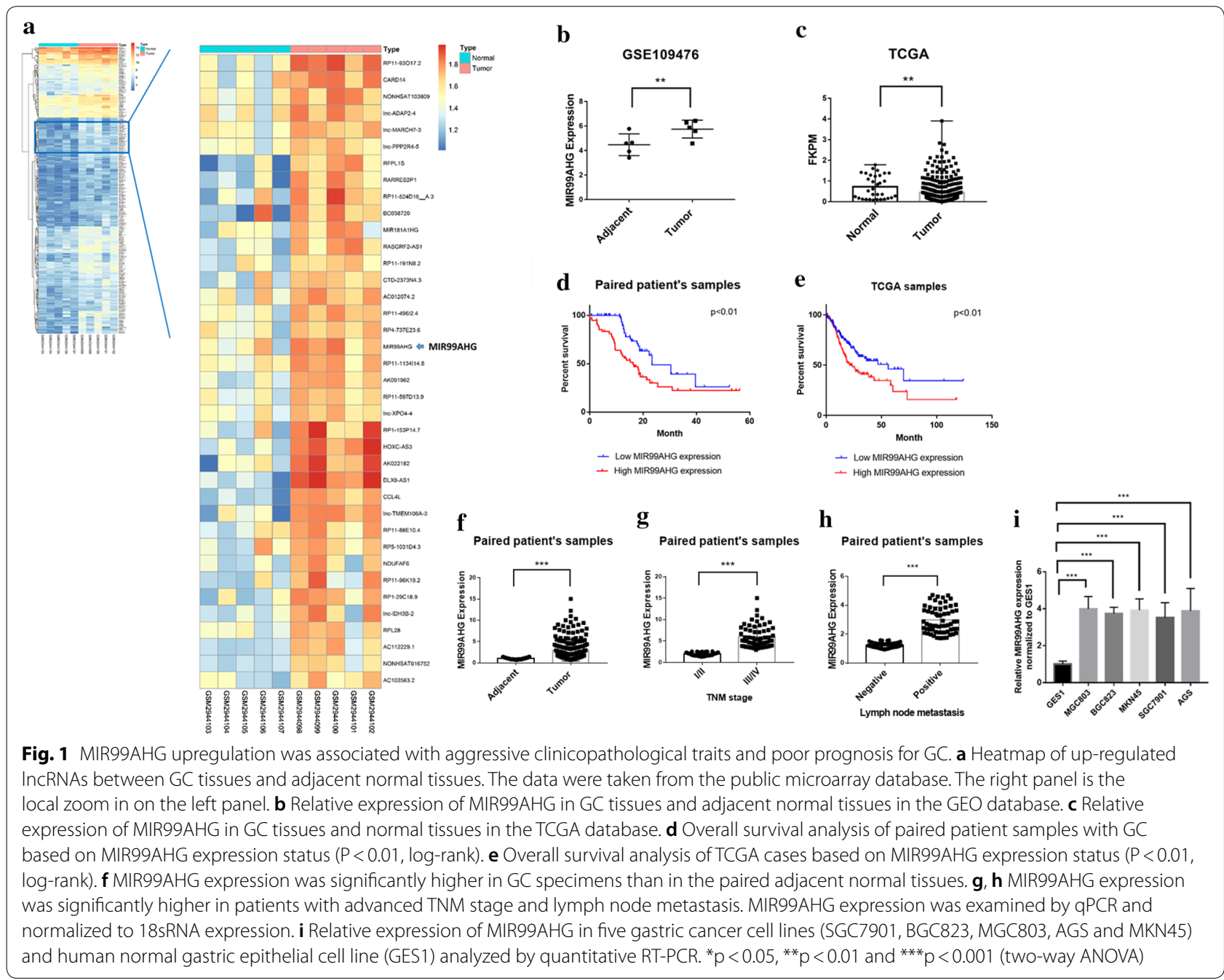


Table 2 Univariate and multivariate Cox regression analysis for OS of patients in study cohort $(n=118)$

\begin{tabular}{|c|c|c|c|}
\hline \multirow[t]{2}{*}{ Variables } & \multicolumn{3}{|l|}{ os } \\
\hline & $\mathrm{HR}$ & $95 \% \mathrm{Cl}$ & $P$ value \\
\hline \multicolumn{4}{|l|}{ Univariate analysis } \\
\hline Age ( $\leq 50$ years vs. $>50$ years $)$ & 0.774 & $0.418-1.490$ & 0.588 \\
\hline Gender (male vs. female) & 1.265 & $0.891-1.608$ & 0.346 \\
\hline Location (distal vs. middle + proximal) & 0.910 & $0.408-1.469$ & 0.577 \\
\hline Tumor size ( $>5 \mathrm{~cm}$ vs. $\leq 5 \mathrm{~cm}$ ) & 1.266 & $0.846-1.794$ & 0.706 \\
\hline $\begin{array}{l}\text { Histologic differentiation } \\
\text { (poorly + undifferentiated vs. } \\
\text { well + moderately) }\end{array}$ & 1.640 & $0.848-2.426$ & 0.178 \\
\hline Invasion depth (T3+T4 vs. T1 + T2) & 2.118 & $1.146-3.312$ & 0.014 \\
\hline TNM stage (III + IV vs. I+ II) & 3.098 & $1.661-4.849$ & 0.007 \\
\hline Lymphatic metastasis (yes vs. no) & 1.211 & $0.594-1.459$ & 0.057 \\
\hline Distant metastasis (yes vs. no) & 1.298 & $0.792-1.899$ & 0.030 \\
\hline Expression of MIR99AHG (high vs. low) & 1.473 & $1.287-2.054$ & 0.001 \\
\hline \multicolumn{4}{|l|}{ Multivariate analysis } \\
\hline TNM stage (III + IV vs. I+ II) & 2.699 & $1.401-4.984$ & 0.006 \\
\hline Invasion depth (T3 + T4 vs. T1 + T2) & 1.121 & $0.504-1.683$ & 0.134 \\
\hline Lymphatic metastasis (yes vs. no) & 1.073 & $0.691-1.662$ & 0.455 \\
\hline Distant metastasis (yes vs. no) & 0.998 & $0.701-1.492$ & 0.074 \\
\hline $\begin{array}{l}\text { Expression of RP11-66B24.7 (high vs. } \\
\text { low) }\end{array}$ & 2.851 & $1.044-4.630$ & 0.035 \\
\hline
\end{tabular}

analyses of predictive clinical variants for survival were shown in Table 2. Then we analyzed the relevance MIR99AHG expression with OS of GC patients in 413 cases from TCGA database. Among those cases, patients were grouped into those with above-median MIR99AHG expression and below-median MIR99AHG expression. Below-median MIR99AHG expression was associated with inferior OS (median OS: 21 months) compared with the above-median (median OS: 46 months; $\mathrm{P}=0.0144$ ) (Fig. 1e). The 3-year OS was $41.45 \%(33.6-49.8 \%)$ in the above-median MIR99AHG expression group and 55.97\% (47.5-62.8\%) in the below-median MIR99AHG expression group. Then expression levels of MIR99AHG were determined by qPCR in 118 paired GC and adjacent nontumor tissues. Expression levels of MIR99AHG in tumor tissues were remarkably higher than that in adjacent tissues (Fig. 2f). Further analyses of the paired patient's samples revealed that MIR99AHG expression in GC tissues was positively related to advanced TNM stage and lymph node metastasis (Fig. 1g, h). The MIR99AHG high group $(\mathrm{n}=59)$ showed poorer histologic differentiation $(P=0.008$, Table 1$)$, higher invasion depth $(P<0.001)$, advanced TNM stage $(P=0.003)$, significantly increased lymph nodes metastasis $(\mathrm{P}<0.001)$ and higher distant metastasis risk $(\mathrm{P}=0.02)$ than the MIR99AHG low group $(n=59)$. There showed no significant correlation between

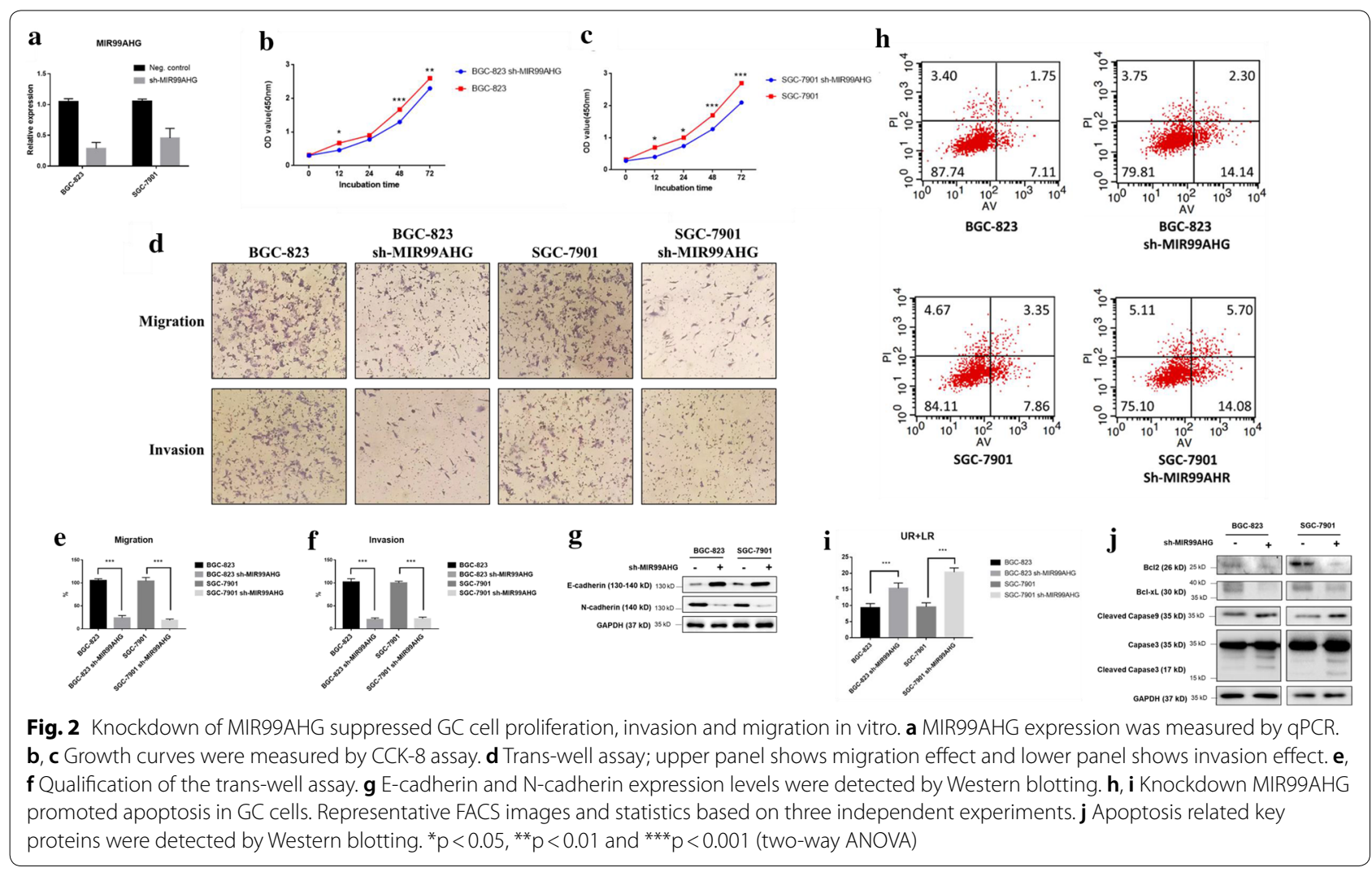




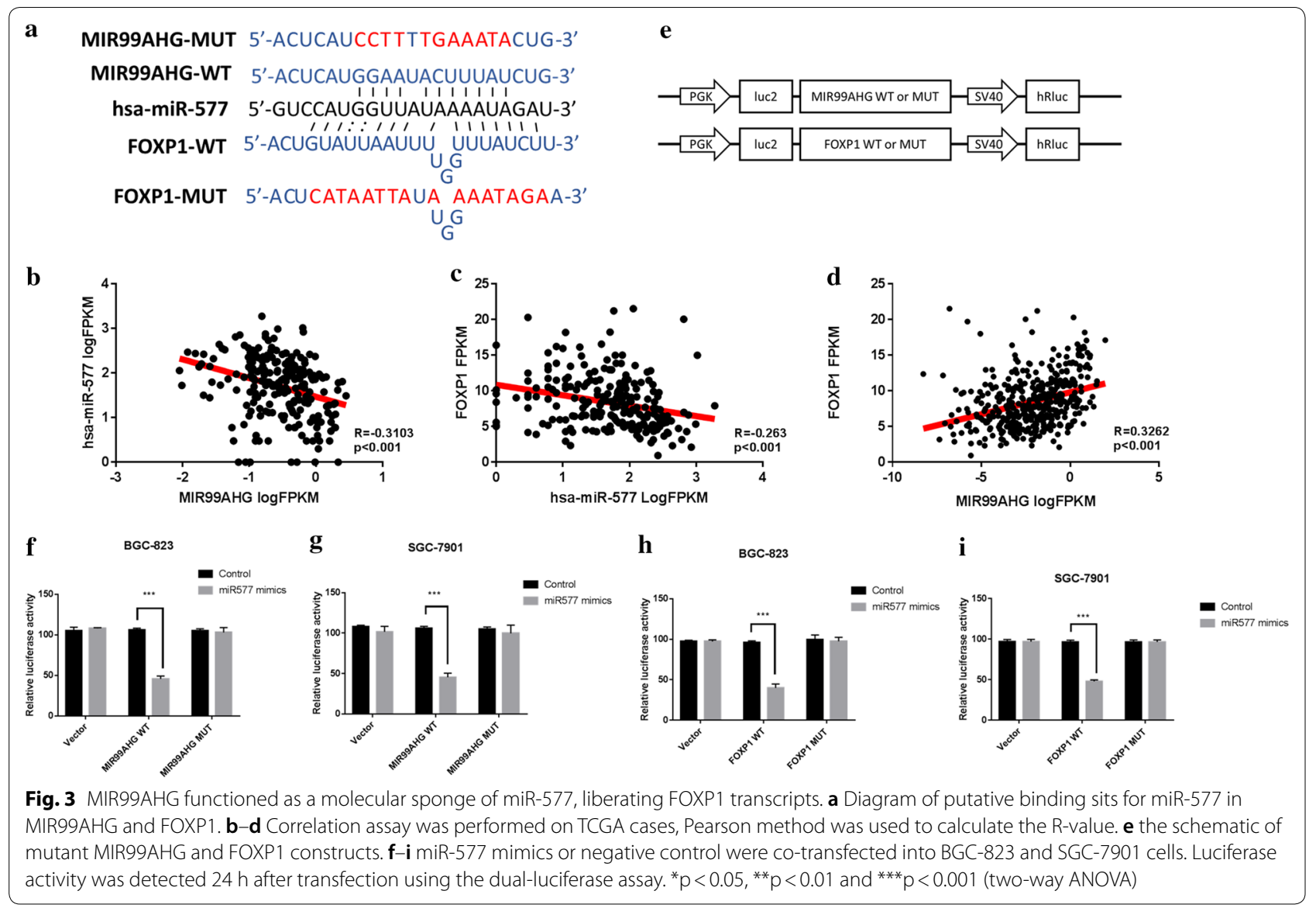

MIR99AHG expression and gender, age, tumor location, and tumor size $(\mathrm{P}>0.05)$. In addition, $\mathrm{qPCR}$ assays were carried out to determine the expression of MIR99AHG in 4 GC cell lines, namely MGC803, BGC823, MKN45, SGC7901, and AGS. MIR99AHG was found to be highly expressed in all the $4 \mathrm{GC}$ cell lines in comparison to GC cell lines (Fig. 1i).

Knockdown of MIR99AHG suppressed GC cell proliferation, invasion, migration and promoted the apoptosis of GC cells in vitro

In order to explore the function of MIR99AHG in GC, we knocked down the MIR99AHG in BGC-823 and SGC-7901 cells by using the RNA interference technique (RNAi). The shRNA showed a good knockdown effect of MIR99AHG (Fig. 2a). Then CCK-8 assays were carried out to detect the role of MIR99AHG on GC cell proliferative ability. The CCK- 8 results showed that knockdown of MIR99AHG reduced the cell proliferative ability of BGC-823 and SGC-7901 (Fig. 2b, c). Trans-well assay results revealed that knockdown of MIR99AHG impaired the invasion and migration of GC cells (Fig. $2 d-f)$. Then we detected molecular markers of EMT in the protein level following MIR99AHG knockdown. The expression of E-cadherin was upregulated while $\mathrm{N}$-cadherin was downregulated when MIR99AHG was knockdown (Fig. 2g). Flow cytometry analysis revealed that MIR99AHG knockdown further promoted the apoptotic rates (early + late-stage apoptosis) of GC compared with the control group (Fig. 2h, i). Western blot analysis demonstrated that MIR99AHG knockdown group showed downregulated expression of anti-apoptotic proteins $\mathrm{Bcl}-2$ and $\mathrm{Bcl}-\mathrm{xl}$ and upregulated expression of pro-apoptotic proteins cleaved caspase- 3 and caspase- 9 (Fig. 2j). These results suggested that knockdown of MIR99AHG may suppress GC cell proliferation, invasion, migration and promote the apoptosis of GC cells.

\section{MIR99AHG/miR-577/FOXP1 axis regulated the malignant phenotype of GC cells}

Researches have revealed that IncRNAs can act as competing endogenous RNAs (ceRNA) for miRNAs. The potential miRNAs that might be sponged by MIR99AHG were predicted through starBase tool (https://starbase.sysu.edu.cn). Then we identified MIR99AHG might sponge to miR-577. Additionally, 
TargetScan analysis predicted the target region for miR-577 in the $3^{\prime}$-UTR of the FOXP1 gene (Fig. 3a), suggesting that miR-577 may directly target FOXP1. Thus, we hypothesized that MIR99AHG might promote FOXP1 expression by interacting with miR-577. Through correlation analysis on TCGA cases, we confirmed that miR-577 expression was negatively correlated with MIR99AHG expression (Fig. 3b). In addition, FOXP1 expression was negatively regulated by miR-577 (Fig. 3c). Then, as expected, expression of MIR99AHG showed a positive correlation with FOXP1 (Fig. 3d). To further validate miR-577 directly binding to FOXP1, the dual-luciferase assay was conducted in GC cell lines. Luciferase vectors were constructed as shown in the scheme (Fig. 3e). We co-transfected the miR-577 mimics and wild type MIR99AHG vector or mutant MIR99AHG vector into GC cells. Results showed that wild type MIR99AHG vector co-transfected cells exhibited lower luciferase activity (Fig. 3f, g). Consistently, miR-577 remarkably decreased the luciferase activity of wild type FOXP1 reporter but not mutated vector (Fig. 3h, i).

\section{MIR99AHG/miR-577/FOXP1 axis regulated the malignant phenotype of GC cells}

Knockdown of MIR99AHG markedly suppressed FOXP1 expression in both BGC-823 and SGC-7901 cells, whereas overexpression of MIR99AHG significantly upregulated FOXP1 expression levels, in both mRNA and protein levels (Fig. 4a, b). In addition, as expected directly FOXP1 knockdown or miR-577 mimics transfection significantly decreased FOXP1 expression (Fig. 4a, b). MIR99AHG overexpression significantly promoted the proliferation and invasion of GC cells, while this effect was abolished by knockdown of FOXP1 (Fig. 4c-g). Western blot results showed that MIR99AHG overexpression significantly downregulated E-cadherin expression and upregulated $\mathrm{N}$-cadherin expression, however knockdown FOXP1 partially reversed this effect (Fig. 4h). These results indicated that FOXP1 could also be regulated by MIR99AHG. MIR99AHG/miR-577/FOXP1 axis regulated migration and invasion ability of GC cells.

\section{FOXP1 potentiated Wnt/ $\beta$-catenin signaling}

It has been reported that FOXP1 overexpression potentiated $\mathrm{Wnt} / \beta$-catenin signaling in diverse cancer cell types [10]. FOXP1 co-complexed with a $\beta$-catenin transcriptional complex on chromatin promoted CBP

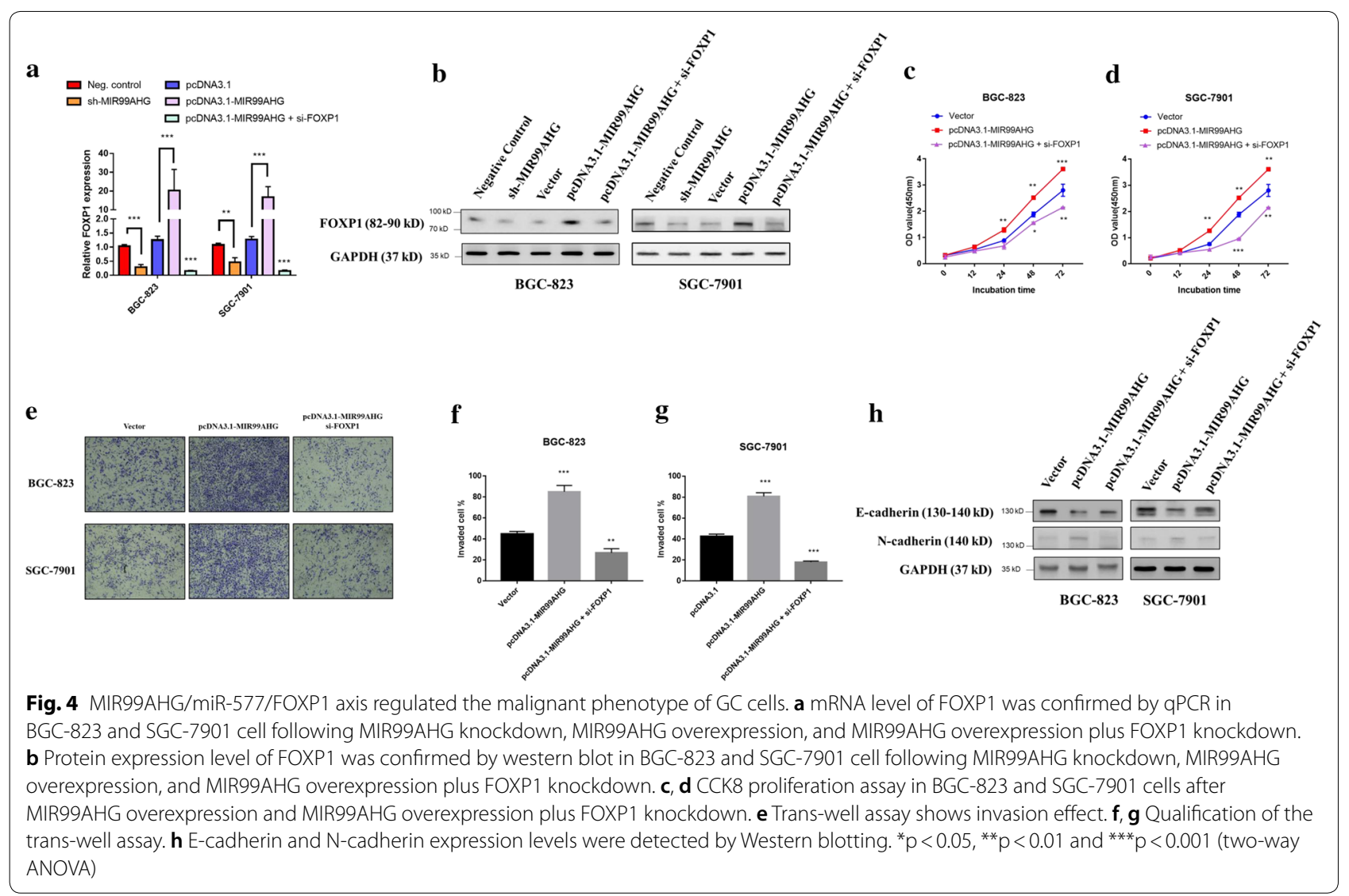


[CREB (adenosine $3^{\prime}, 5^{\prime}$-monophosphate response element-binding protein)-binding protein]-dependent acetylation of $\beta$-catenin, resulting in enhanced $\beta$-catenin-dependent transcription $[10,11]$. Therefore, we tested whether MIR99AHG promotes FOXP1 meditated acetylation of $\beta$-catenin at Lys49. Indeed, MIR99AHG overexpression induced acetylation of Lys49 in $\beta$-catenin and the acetylation was suppressed by siRNA-mediated silencing of FOXP1 (Fig. 5a). In addition, overexpression of MIR99AHG promoted $\beta$-catenin nuclear transportation, and the MIR99AHG mediated $\beta$-catenin nuclear transportation abolished by FOXP1 knockdown (Fig. 5b). The immunocytochemistry (ICC) results showed that MIR99AHG overexpression stabilized $\beta$-catenin and the stabilized $\beta$-catenin mainly located in the nuclear, however the stabilization phenomena was abolished by FOXP1 knockdown (Fig. 5c).

\section{Discussion}

Gastric cancer is the fifth most common malignancy with a third highest cancer associated death rate [12]. Recently, various studies have suggested that lncRNAs are closely related to the carcinogenesis and development of GC [13-17]. LncRNA TINCR could promote the metastatic ability of GC cells by regulating the stability of KLF2 [18, 19]. LncRNA SNHG16 was reported to promote the proliferation, invasion and apoptosis of GC by sponging miR-135, thus activating JAK2/STAT3 pathway [20]. Moreover, IncRNA MALAT1 and MACC1-AS1was demonstrated to regulate the chemoresistance of GC $[16,21]$. Therefore, exploring the roles and mechanisms of lncRNAs might shed new light on the diagnosis and treatment of GC $[22,23]$.

Here we elucidated the aberrant upregulation of MIR99AHG in GC. Firstly, we downloaded and analyzed previous microarray datasets (GSE109476) to explore the different lncRNA expression patterns between cancer

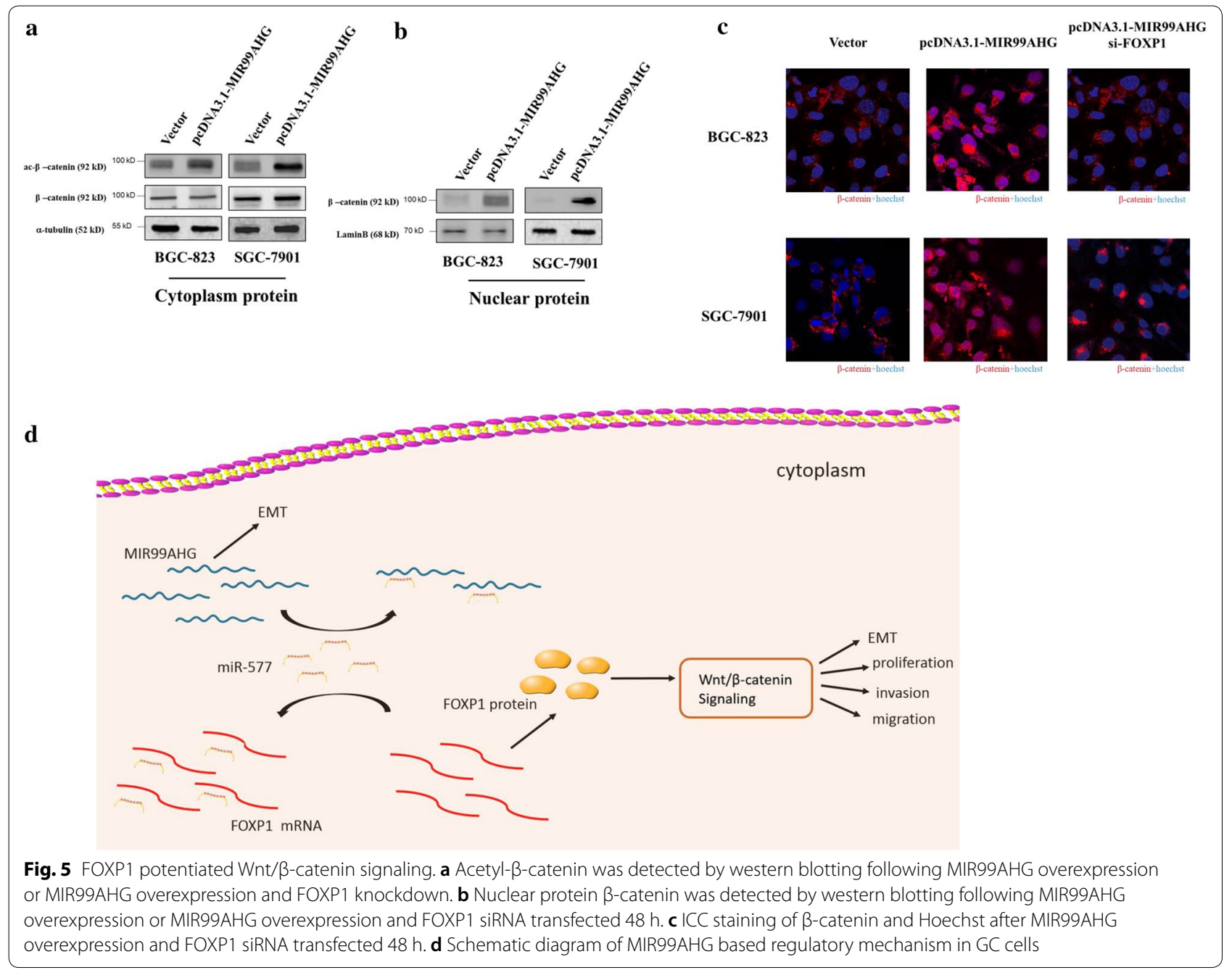


and adjuvant normal tissues. We found that MIR99AHG was significantly upregulated in GC cancer tissues. To further investigate the expression difference, we compared MIR99AHG expression levels in the patients from the TCGA database. Similarly, MIR99AHG expression levels were higher and showed broader ranging in GC group compared with normal group. Later, we investigated 118 paired GC cancer tissues and non-cancer tissues. The survival curve revealed that the MIR99AHG level was negatively related to the OS of GC. The correlation between MIR99AHG expression and clinicopathological features of GC patients was further explored. The results showed that expression of MIR99AHG was positively associated to TNM stage and lymph node metastasis (Table 2). Subsequent experiments showed that knockdown of MIR99AHG suppressed the proliferation and invasion of the GC cells by regulating EMT and meanwhile, induced apoptosis progression.

One of the important roles of lncRNAs is to act as ceRNAs by sponging to miRNAs. For example, lncRNA MIR99AHG promoted apoptosis of neuroblastoma cells by targeting miR-296-5p [24]. FOXP1, fork head box protein 1 , has been demonstrated to act as a $\mathrm{B}$ cell oncogene and was involved in B-cell differentiation and survival $[25,26]$. FOXP1 was positively associated with the expression of BCL2 and might affect cell apoptosis [27, 28]. Based on our analysis, FOXP1 expression was negatively regulated by miR-577 while MIR99AHG showed a positive relation with FOXP1. Further experiments then showed that knockdown of FOXP1 or miR-577 mimics transfection could abolish the effect of MIR99AHG on cell proliferation and migration. FOXP1 co-complexed with a $\beta$-catenin transcriptional complex on chromatin promoted CBP-dependent acetylation of $\beta$-catenin, resulting in enhanced $\beta$-catenin-dependent transcription. Our results depicted that MIR99AHG overexpression stabilized $\beta$-catenin and the stabilized $\beta$-catenin mainly located in the nuclear, however the stabilization phenomena was abolished by FOXP1 knockdown. Take together, our data suggested that MIR99AHG overexpression could sponge to miR-577, which led to an increase of FOXP1 expression. Then, overexpressed FOXP1 caused $\beta$-catenin abnormal acetylation and resulted in WNT signaling abnormal activation.

\section{Conclusions}

Taken together, we illustrated that lncRNA MIR99AHG was highly expressed in both GC tissues and cell lines. MIR99AHG could act as a ceRNA for miR-577, thus activating the FOXP1 mediated Wnt/ $\beta$-catenin pathway. Moreover, a higher level of MIR99AHG was correlated with more advanced tumor progression and poorer prognosis. Our data that MIR99AHG might be useful as a diagnostic and prognostic biomarker as well as a therapeutic target for gastric cancer in the future.

\section{Abbreviations}

AMKL: Acute megakaryoblastic leukemia; AML: Acute myeloid leukemia; ceRNA: Competing for endogenous RNA; FBS: Fetal bovine serum; FITC: Fluorescein isothiocyanate; GC: Gastric cancer; GEO: Gene Expression Omnibus; IncRNAs: Long non-coding RNAs; miRNAs: Acute megakaryoblastic leukemia; OD: Optical density; PVDF: Polyvinylidene fluoride; qRT-PCR: Quantitative realtime PCR; SDS-PAGE: Sodium dodecyl sulfate-polyacrylamide gel electrophoresis; TCGA: The Cancer Genome Atlas.

\section{Acknowledgements}

None.

\section{Authors' contributions}

$\mathrm{QM}, \mathrm{XW}$ and $\mathrm{KZ}$ designed the study; QM, XW, TX and QZ performed the experiments; $K Z$ supervised the project; $Q M$ and XW analysed the data; QM and XW drafted the paper. All authors read and approved the final manuscript.

\section{Funding}

This research did not receive any specific grant.

\section{Availability of data and materials}

Data and materials supporting the current study are all available from the corresponding author on reasonable request.

\section{Ethics approval and consent to participate}

The use of the patient-derived samples from 118 patients was approved by the Institutional Review Board of the Zhongshan Hospital, Fudan University. Informed consent was obtained from all patients. This study was conducted in accordance with the Declaration of Helsinki.

\section{Consent for publication}

No consent was involved in this publication.

\section{Competing interests}

The authors have no conflicts of interest to disclose.

\section{Author details}

${ }^{1}$ Department of General Surgery, Zhongshan Hospital, Fudan University, Shanghai, China. ${ }^{2}$ Department of General Surgery, The Affiliated Hospital of Yangzhou University, Yangzhou University, Yangzhou, China. ${ }^{3}$ Department of Oncology, Huaian Hospital of Huaian City, No.161 Zhenhuailou East Road, Huai'an 223200, Jiangsu, China.

Received: 19 February 2020 Accepted: 19 August 2020

Published online: 27 August 2020

References

1. Huang KK, Ramnarayanan K, Zhu F, Srivastava S, Xu C, Tan ALK, et al. Genomic and epigenomic profiling of high-risk intestinal metaplasia reveals molecular determinants of progression to gastric cancer. Cancer Cell. 2018:33(1):137-150.e5.

2. Li PF, Chen SC, Xia T, Jiang XM, Shao YF, Xiao BX, et al. Non-coding RNAs and gastric cancer. World J Gastroenterol. 2014;20(18):5411-9.

3. Li Z, Ding Z, Rong D, Tang W, Cao H. Overexpression of IncRNA AFAP1-AS1 promotes cell proliferation and invasion in gastric cancer. Oncol Lett. 2019;18(3):3211-7.

4. Wang CJ, Zhu CC, Xu J, Wang M, Zhao WY, Liu Q, et al. The IncRNA UCA1 promotes proliferation, migration, immune escape and inhibits apoptosis in gastric cancer by sponging anti-tumor miRNAs. Mol Cancer. 2019;18(1):115

5. Tanizawa Y, Terashima M. Lymph node dissection in the resection of gastric cancer: review of existing evidence. Gastric Cancer. 2010;13(3):137-48. 
6. Djebali S, Davis CA, Merkel A, Dobin A, Lassmann T, Mortazavi A, et al. Landscape of transcription in human cells. Nature. 2012;489(7414):101-8.

7. Wang WJ, Guo CA, Li R, Xu ZP, Yu JP, Ye Y, et al. Long non-coding RNA CASC 19 is associated with the progression and prognosis of advanced gastric cancer. Aging. 2019;11(15):5829-47.

8. Chen C, Tang X, Liu Y, Zhu J, Liu J. Induction/reversal of drug resistance in gastric cancer by non-coding RNAs (Review). Int J Oncol. 2019;54(5):1511-24.

9. Emmrich S, Streltsov A, Schmidt F, Thangapandi VR, Reinhardt D, Klusmann JH. LincRNAs MONC and MIR100HG act as oncogenes in acute megakaryoblastic leukemia. Mol Cancer. 2014;13:171.

10. Walker MP, Stopford CM, Cederlund M, Fang F, Jahn C, Rabinowitz AD, et al. FOXP1 potentiates Wnt/beta-catenin signaling in diffuse large B cell lymphoma. Sci Signal. 2015;8(362):ra12.

11. Zaiss DMW, Coffer PJ. Forkhead box transcription factors as contextdependent regulators of lymphocyte homeostasis. Nat Rev Immunol. 2018;18(11):703-15.

12. Zhang P, Li S, Chen Z, Lu Y, Zhang H. LncRNA SNHG8 promotes proliferation and invasion of gastric cancer cells by targeting the miR-491/PDGFRA axis. Hum Cell. 2019:33:123-30.

13. Wang CJ, Zhu CC, Xu J, Wang M, Zhao WY, Liu Q, et al. Correction to: The IncRNA UCA1 promotes proliferation, migration, immune escape and inhibits apoptosis in gastric cancer by sponging anti-tumor miRNAs. Mol Cancer. 2019;18(1):129.

14. Kong R, Zhang EB, Yin DD, You LH, Xu TP, Chen WM, et al. Long noncoding RNA PVT1 indicates a poor prognosis of gastric cancer and promotes cell proliferation through epigenetically regulating p15 and p16. Mol Cancer. 2015;14:82.

15. Sun TT, He J, Liang Q, Ren LL, Yan TT, Yu TC, et al. LncRNA GClnc1 promotes gastric carcinogenesis and may act as a modular scaffold of WDR5 and KAT2A complexes to specify the histone modification pattern. Cancer Discov. 2016;6(7):784-801.

16. He W, Liang B, Wang C, Li S, Zhao Y, Huang Q, et al. MSC-regulated IncRNA MACC1-AS1 promotes stemness and chemoresistance through fatty acid oxidation in gastric cancer. Oncogene. 2019;38(23):4637-54.

17. Wang X, Liang Q, Zhang L, Gou H, Li Z, Chen H, et al. C8orf76 promotes gastric tumorigenicity and metastasis by directly inducing IncRNA DUSP5P1 and associates with patient outcomes. Clin Cancer Res. 2019;25(10):3128-40.

18. Xu TP, Liu XX, Xia R, Yin L, Kong R, Chen WM, et al. SP1-induced upregulation of the long noncoding RNA TINCR regulates cell proliferation and apoptosis by affecting KLF2 mRNA stability in gastric cancer. Oncogene. 2015;34(45):5648-61.

19. Gibb EA, Warren RL, Wilson GW, Brown SD, Robertson GA, Morin GB, et al. Activation of an endogenous retrovirus-associated long non-coding RNA in human adenocarcinoma. Genome Med. 2015;7(1):22.

20. Wang X, Kan J, Han J, Zhang W, Bai L, Wu H. LncRNA SNHG16 functions as an oncogene by sponging MiR-135a and promotes JAK2/STAT3 signal pathway in gastric cancer. J Cancer. 2019;10(4):1013-22.

21. Zhang G, Li S, Lu J, Ge Y, Wang Q, Ma G, et al. LncRNA MT1JP functions as a ceRNA in regulating FBXW7 through competitively binding to miR92a-3p in gastric cancer. Mol Cancer. 2018;17(1):87.

22. Gunther T, Schneider-Stock R, Hackel C, Pross M, Schulz HU, Lippert H, et al. Telomerase activity and expression of hTRT and hTR in gastrointestinal stromal tumors in comparison with extragastrointestinal sarcomas. Clin Cancer Res. 2000;6(5):1811-8.

23. Kim HP, Cho GA, Han SW, Shin JY, Jeong EG, Song SH, et al. Novel fusion transcripts in human gastric cancer revealed by transcriptome analysis. Oncogene. 2014;33(47):5434-41.

24. Johnston TP, Bove EL, Bolling SF, Schoen FJ, Boyd JA, Golomb G, et al. Local controlled release of 1-hydroxyethylidene diphosphonate using silicone-rubber matrices. Effects of sterilization on in vitro release and in vivo efficacy. ASAIO Trans. 1988;34(3):835-8.

25. Hu H, Wang B, Borde M, Nardone J, Maika S, Allred L, et al. Foxp1 is an essential transcriptional regulator of $B$ cell development. Nat Immunol. 2006;7(8):819-26.

26. Craig VJ, Cogliatti SB, Imig J, Renner C, Neuenschwander S, Rehrauer H, et al. Myc-mediated repression of microRNA-34a promotes high-grade transformation of B-cell lymphoma by dysregulation of FoxP1. Blood. 2011;117(23):6227-366

27. Yu B, Zhou X, Li B, Xiao X, Yan S, Shi D. FOXP1 expression and its clinicopathologic significance in nodal and extranodal diffuse large B-cell lymphoma. Ann Hematol. 2011;90(6):701-8.

28. Sagaert $X$, de Paepe $P$, Libbrecht L, Vanhentenrijk V, Verhoef $G$, Thomas J, et al. Forkhead box protein P1 expression in mucosa-associated lymphoid tissue lymphomas predicts poor prognosis and transformation to diffuse large B-cell lymphoma. J Clin Oncol. 2006;24(16):2490-7.

\section{Publisher's Note}

Springer Nature remains neutral with regard to jurisdictional claims in published maps and institutional affiliations.
Ready to submit your research? Choose BMC and benefit from:

- fast, convenient online submission

- thorough peer review by experienced researchers in your field

- rapid publication on acceptance

- support for research data, including large and complex data types

- gold Open Access which fosters wider collaboration and increased citations

- maximum visibility for your research: over 100M website views per year

At $\mathrm{BMC}$, research is always in progress.

Learn more biomedcentral.com/submissions 